\title{
Generalized parton distributions in the deuteron
}

\author{
E. R. Berger ${ }^{1}$, F. Cano ${ }^{2}$, M. Diehl ${ }^{3}$ and B. Pire ${ }^{1}$ \\ ${ }^{1}$ CPhT, École Polytechnique, F-91128 Palaiseau, France \\ ${ }^{2}$ DAPNIA/SPhN, CEA/Saclay, F-91191 Gif sur Yvette Cedex, France \\ ${ }^{3}$ Deutsches Elektronen-Synchroton DESY, D-22603 Hamburg, Germany
}

\begin{abstract}
We introduce generalized quark and gluon distributions in the deuteron, which can be measured in exclusive processes like deeply virtual Compton scattering and meson electroproduction. We discuss the basic properties of these distributions, and point out how they probe the interplay of nucleon and parton degrees of freedom in the deuteron wave function.
\end{abstract}

Introduction. The partonic structure of the deuteron has been explored in terms of the parton distributions accessible in deep inelastic scattering [1], and in terms of the form factors measured in elastic lepton-deuteron processes [2,3]. It is natural to ask what can be learned from generalized parton distributions (GPDs), introduced not long ago in [4,5]. For the nucleon case it has been shown that these quantities contain unique information about the dynamics of quarks and gluons in QCD bound states, beyond what can be unraveled from ordinary parton distributions and form factors. Here we extend these studies to the case of the deuteron, with the aim of providing the theoretical framework to analyze and interpret present and future measurements with deuteron targets. We restrict ourselves to parton distributions of twist two, and to the parton helicity conserving sector, which is relevant in most phenomenological applications. Quark and gluon helicity flip GPDs can be treated with the same methods.

Generalized quark distributions for the deuteron. As in the nucleon case, the GPDs for the deuteron are defined through non-diagonal matrix elements of quarkantiquark operators on the light cone. Their general decomposition can be written as

$$
\begin{aligned}
V_{\lambda^{\prime} \lambda} & =\int \frac{d \kappa}{2 \pi} e^{i x \kappa P . n}\left\langle p^{\prime}, \lambda^{\prime}|\bar{\psi}(-\kappa n) \gamma \cdot n \psi(\kappa n)| p, \lambda\right\rangle \\
& =\sum_{i} \epsilon^{* \beta} V_{\beta \alpha}^{(i)} \epsilon^{\alpha} H_{i}(x, \xi, t) \\
A_{\lambda^{\prime} \lambda} & =\int \frac{d \kappa}{2 \pi} e^{i x \kappa P \cdot n}\left\langle p^{\prime}, \lambda^{\prime}\left|\bar{\psi}(-\kappa n) \gamma \cdot n \gamma_{5} \psi(\kappa n)\right| p, \lambda\right\rangle \\
& =\sum_{i} \epsilon^{* \beta} A_{\beta \alpha}^{(i)} \epsilon^{\alpha} \tilde{H}_{i}(x, \xi, t)
\end{aligned}
$$

where $n$ is a light-like four-vector, $n^{2}=0$. The incoming and outgoing deuterons respectively have momenta $p, p^{\prime}$, helicities $\lambda, \lambda^{\prime}$, and polarization vectors $\epsilon=\epsilon(p, \lambda)$ and $\epsilon^{\prime}=\epsilon^{\prime}\left(p^{\prime}, \lambda^{\prime}\right)$. We write $P=p+p^{\prime}$ and $\Delta=p^{\prime}-$ $p$, and choose Ji's variables $x, \xi=-(\Delta . n) /(P . n), t=$ $\Delta^{2}$ as arguments of the GPDs $H_{i}$ and $\tilde{H}_{i}$. The tensors $V^{(i)}$ and $A^{(i)}$ depend on the four-vectors $p, p^{\prime}$, and $n$. One need only keep tensors which do not vanish when contracted with $\epsilon_{\alpha}, \epsilon_{\beta}^{\prime}$, given the orthogonality conditions $\epsilon \cdot p=\epsilon^{\prime} \cdot p^{\prime}=0$ of the polarization vectors. With the constraints from parity invariance we find that the $V_{\beta \alpha}^{(i)}$ are linear combinations of the five tensor structures

$$
\left\{g_{\beta \alpha}, p_{\beta} n_{\alpha}, n_{\beta} p_{\alpha}^{\prime}, p_{\beta} p_{\alpha}^{\prime}, n_{\beta} n_{\alpha}\right\}
$$

Similarly, the $A_{\beta \alpha}^{(i)}$ are linear combinations of the seven tensors

$$
\begin{aligned}
& \left\{\epsilon_{\mu \nu \beta \alpha} p^{\mu} p^{\prime \nu}, \epsilon_{\mu \nu \beta \alpha} n^{\mu} p^{\nu}, \epsilon_{\mu \nu \beta \alpha} n^{\mu} p^{\prime \nu}\right. \\
& \epsilon_{\mu \nu \rho \beta} p^{\mu} p^{\prime \nu} n^{\rho} n_{\alpha}, \epsilon_{\mu \nu \rho \beta} p^{\mu} p^{\prime \nu} n^{\rho} p_{\alpha}^{\prime} \\
& \left.\epsilon_{\mu \nu \rho \alpha} p^{\mu} p^{\prime \nu} n^{\rho} n_{\beta}, \epsilon_{\mu \nu \rho \alpha} p^{\mu} p^{\prime \nu} n^{\rho} p_{\beta}\right\} .
\end{aligned}
$$

Using the Schouten identities [6] one can show that only four out of these seven are linearly independent. The first $x$ moments of generalized parton distributions are elastic form factors. To keep the corresponding relations simple we take among the tensors $V^{(i)}, A^{(i)}$ those which appear in the conventional form factor decomposition of the vector and axial currents [7]:

$$
\begin{aligned}
& \left\langle p^{\prime}, \lambda^{\prime}\left|\bar{\psi}(0) \gamma^{\mu} \psi(0)\right| p, \lambda\right\rangle=-G_{1}(t)\left(\epsilon^{\prime *} . \epsilon\right) P^{\mu} \\
& \quad+G_{2}(t)\left[\epsilon^{\mu}\left(\epsilon^{\prime *} . P\right)+\epsilon^{\prime * \mu}(\epsilon . P)\right] \\
& \quad-G_{3}(t)(\epsilon . P)\left(\epsilon^{* *} . P\right) \frac{P^{\mu}}{2 M^{2}}, \\
& \left\langle p^{\prime}, \lambda^{\prime}\left|\bar{\psi}(0) \gamma^{\mu} \gamma_{5} \psi(0)\right| p, \lambda\right\rangle=-i \tilde{G}_{1}(t) \epsilon^{\mu}{ }_{\alpha \beta \gamma} \epsilon^{\prime * \alpha} \epsilon^{\beta} P^{\gamma} \\
& \quad+i \tilde{G}_{2}(t) \epsilon_{\alpha \beta \gamma}^{\mu} \Delta^{\alpha} P^{\beta} \frac{\epsilon^{\gamma}\left(\epsilon^{\prime *} . P\right)+\epsilon^{\prime * \gamma}(\epsilon . P)}{M^{2}}
\end{aligned}
$$

where our convention for the antisymmetric tensor is $\epsilon_{0123}=1$ and $\mathrm{M}$ is the deuteron mass. The matrix elements are here defined flavor by flavor; to get the conventional form factors, one must weight with electromagnetic or weak charges and sum over flavors. For the matrix elements of the non-local operators we define 


$$
\begin{aligned}
& V_{\lambda^{\prime} \lambda}=-\left(\epsilon^{\prime *} . \epsilon\right) H_{1}+\frac{(\epsilon . n)\left(\epsilon^{\prime *} . P\right)+\left(\epsilon^{\prime *} . n\right)(\epsilon . P)}{P . n} H_{2} \\
& -\frac{(\epsilon . P)\left(\epsilon^{\prime *} . P\right)}{2 M^{2}} H_{3}+\frac{(\epsilon . n)\left(\epsilon^{* *} . P\right)-\left(\epsilon^{\prime *} . n\right)(\epsilon . P)}{P . n} H_{4} \\
& +\left\{4 M^{2} \frac{(\epsilon . n)\left(\epsilon^{\prime *} . n\right)}{(P . n)^{2}}+\frac{1}{3}\left(\epsilon^{\prime *} . \epsilon\right)\right\} H_{5} \\
& A_{\lambda^{\prime} \lambda}=-i \frac{\epsilon_{\mu \alpha \beta \gamma} n^{\mu} \epsilon^{\prime * \alpha} \epsilon^{\beta} P^{\gamma}}{P . n} \tilde{H}_{1} \\
& +i \frac{\epsilon_{\mu \alpha \beta \gamma} n^{\mu} \Delta^{\alpha} P^{\beta}}{P . n} \frac{\epsilon^{\gamma}\left(\epsilon^{\prime *} . P\right)+\epsilon^{\prime * \gamma}(\epsilon . P)}{M^{2}} \tilde{H}_{2} \\
& +i \frac{\epsilon_{\mu \alpha \beta \gamma} n^{\mu} \Delta^{\alpha} P^{\beta}}{P . n} \frac{\epsilon^{\gamma}\left(\epsilon^{\prime *} . P\right)-\epsilon^{\prime * \gamma}(\epsilon . P)}{M^{2}} \tilde{H}_{3} \\
& +i \frac{\epsilon_{\mu \alpha \beta \gamma} n^{\mu} \Delta^{\alpha} P^{\beta}}{P . n} \frac{\epsilon^{\gamma}\left(\epsilon^{\prime *} . n\right)+\epsilon^{\prime * \gamma}(\epsilon . n)}{P . n} \tilde{H}_{4} .
\end{aligned}
$$

Since it is determined by the quark operators in Eqs. (11) and (2), the $Q^{2}$ evolution of the GPDs $H_{i}$ and $\tilde{H}_{i}$ is exactly the same as for spin $1 / 2$ targets, worked out in

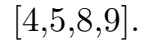

Time reversal properties and sum rules. The action of the time reversal operator on the matrix element (1) leads to the relation

$$
\begin{aligned}
& \epsilon^{* \beta} \epsilon^{\alpha} V_{\beta \alpha}^{(i)}(P, \Delta, n) H_{i}(x, \xi, t) \\
= & \epsilon^{* \beta} \epsilon^{\prime \alpha} V_{\beta \alpha}^{(i)}(P,-\Delta, n) H_{i}(x,-\xi, t),
\end{aligned}
$$

where we have made explicit the dependence of the tensors $V^{(i)}$ on the relevant four-vectors. Taking the complex conjugate of Eq. (11) we get

$$
\begin{aligned}
& \epsilon^{\prime \beta} \epsilon^{* \alpha} V_{\beta \alpha}^{*(i)}(P, \Delta, n) H_{i}^{*}(x, \xi, t) \\
= & \epsilon^{* \beta} \epsilon^{\prime \alpha} V_{\beta \alpha}^{(i)}(P,-\Delta, n) H_{i}(x,-\xi, t) .
\end{aligned}
$$

For the axial vector case, we obtain relations analogous to (7) and (8) by replacing $V^{(i)}$ with $A^{(i)}$ and $H_{i}$ with $\tilde{H}_{i}$. Combining these conditions we find that all nine GPDs are real. However, their behavior under time reversal is not uniform and we have:

$$
\begin{aligned}
& H_{i}(x, \xi, t)=H_{i}(x,-\xi, t) \quad(i=1,2,3,5), \\
& H_{4}(x, \xi, t)=-H_{4}(x,-\xi, t), \\
& \tilde{H}_{i}(x, \xi, t)=\tilde{H}_{i}(x,-\xi, t) \quad(i=1,2,4), \\
& \tilde{H}_{3}(x, \xi, t)=-\tilde{H}_{3}(x,-\xi, t) .
\end{aligned}
$$

Note that in the non-forward case, time reversal invariance fixes the phase of the generalized parton distributions and determines their behavior under sign change of the skewedness parameter $\xi$, but does not limit the number of GPDs 10]. Integrating $V_{\lambda^{\prime} \lambda}$ and $A_{\lambda^{\prime} \lambda}$ over $x$ one obtains the local matrix elements (5) contracted with $n^{\mu} /(P . n)$. Since the tensors that accompany the distributions $H_{i}, \tilde{H}_{i}$ in Eq. (6) are linearly independent, we obtain the sum rules

$$
\begin{array}{ll}
\int_{-1}^{1} d x H_{i}(x, \xi, t)=G_{i}(t) & (i=1,2,3), \\
\int_{-1}^{1} d x \tilde{H}_{i}(x, \xi, t)=\tilde{G}_{i}(t) & (i=1,2), \\
\int_{-1}^{1} d x H_{4}(x, \xi, t)=\int_{-1}^{1} d x \tilde{H}_{3}(x, \xi, t)=0 \\
\int_{-1}^{1} d x H_{5}(x, \xi, t)=\int_{-1}^{1} d x \tilde{H}_{4}(x, \xi, t)=0 .
\end{array}
$$

The integrals over $H_{4}, \tilde{H}_{3}$ and $H_{5}, \tilde{H}_{4}$ do not correspond to form factors of the local vector or axial currents and therefore vanish. In the case of $H_{4}$ and $\tilde{H}_{3}$ this is due to time reversal constraints, whereas the definitions of $H_{5}$ and $\tilde{H}_{4}$ involve the tensor $n^{\mu} n^{\nu} /(P . n)^{2}$, whose analog cannot appear in the decomposition of the local currents due to Lorentz invariance.

The forward limit. Let now study the forward limit of the GPDs, which defines the usual parton distributions. In the parton model, i.e., at leading twist and leading order in $\alpha_{s}$ there are three independent structure functions in deep inelastic scattering, $F_{1}, b_{1}, g_{1}$, whose probabilistic interpretation in terms of quark densities reads [1]

$$
\begin{aligned}
& F_{1}(x)=\frac{1}{2} \sum_{q} e_{q}^{2} \frac{q^{1}(x)+q^{-1}(x)+q^{0}(x)}{3}+\{q \rightarrow \bar{q}\} \\
& b_{1}(x)=\frac{1}{2} \sum_{q} e_{q}^{2}\left[q^{0}(x)-\frac{q^{1}(x)+q^{-1}(x)}{2}\right]+\{q \rightarrow \bar{q}\} \\
& g_{1}(x)=\frac{1}{2} \sum_{q} e_{q}^{2}\left[q_{\uparrow}^{1}(x)-q_{\uparrow}^{-1}(x)\right]+\{q \rightarrow \bar{q}\}
\end{aligned}
$$

Here $q_{\uparrow(\downarrow)}^{\lambda}(x)$ represents the probability to find a quark with momentum fraction $x$ and positive (negative) helicity in a deuteron target of helicity $\lambda$. The unpolarized quark densities $q^{\lambda}$ are defined as $q^{\lambda}(x)=q_{\uparrow}^{\lambda}(x)+q_{\jmath}^{\lambda}(x)$. From parity one has $q_{\uparrow}^{\lambda}=q_{\downarrow}^{-\lambda}$. The densities for antiquarks are defined in analogy. Note that the probabilistic interpretation for $F_{1}$ and $g_{1}$ is similar to the one in the spin $1 / 2$ case, whereas the function $b_{1}$ does not appear for spin $1 / 2$ targets. In the forward limit the only structures in Eq. (6) that survive are those proportional to $H_{1}, H_{5}$ and $\tilde{H}_{1}$, because in that limit we have $\Delta=0$ and $\epsilon \cdot P=\epsilon^{\prime} . P=0$. Using the results for helicity amplitudes given below, one gets:

$$
\begin{aligned}
& H_{1}(x, 0,0)=\frac{q^{1}(x)+q^{-1}(x)+q^{0}(x)}{3}, \\
& H_{5}(x, 0,0)=q^{0}(x)-\frac{q^{1}(x)+q^{-1}(x)}{2}, \\
& \tilde{H}_{1}(x, 0,0)=q_{\uparrow}^{1}(x)-q_{\uparrow}^{-1}(x)
\end{aligned}
$$

for $x>0$. The corresponding relations for $x<0$ involve the antiquark distributions at $-x$, with an overall minus sign in the expressions for $H_{1}$ and $H_{5}$. With Eq. (10) we thus have 


$$
\begin{aligned}
0 & =\int_{-1}^{1} d x H_{5}(x, 0,0) \\
& =\int_{0}^{1} d x\left[q^{0}(x)-\frac{q^{1}(x)+q^{-1}(x)}{2}\right]-\{q \rightarrow \bar{q}\}
\end{aligned}
$$

and recover the parton model sum rule $\int_{0}^{1} b_{1}(x)=0$ of [11], which was obtained under the assumption that the quark sea $q-\bar{q}$ does not contribute to this integral.

Helicity amplitudes. In the region $\xi<x<1$ our GPDs can be represented in terms of amplitudes for the scattering of a quark on a deuteron 10], defined as

$$
\mathcal{A}_{\lambda^{\prime} \pm, \lambda \pm}=\frac{1}{2}\left(V_{\lambda^{\prime} \lambda} \pm A_{\lambda^{\prime} \lambda}\right)
$$

with \pm referring to the helicities of the quarks. With the constraints

$$
\mathcal{A}_{-\lambda^{\prime}-\mu,-\lambda-\mu}=(-1)^{\lambda^{\prime}-\lambda} \mathcal{A}_{\lambda^{\prime} \mu, \lambda \mu}
$$

from parity invariance there are nine independent quark helicity conserving amplitudes. Since $\mathcal{A}_{0+, 0+}=\mathcal{A}_{0-, 0-}$ we have only four quark helicity dependent distributions $\tilde{H}_{i}$, compared with the five quark helicity independent $H_{i}$. Time reversal invariance gives

$$
\mathcal{A}(x, \xi, t)_{\lambda \mu, \lambda^{\prime} \mu}=(-1)^{\lambda^{\prime}-\lambda} \mathcal{A}(x,-\xi, t)_{\lambda^{\prime} \mu, \lambda \mu},
$$

and thus does not further reduce the number of GPDs, as remarked above. To define the polarization of the incoming deuteron we introduce

$$
\begin{aligned}
& \epsilon^{(0) \mu}= \frac{1}{M}\left(p^{\mu}-\frac{2 M^{2}}{1+\xi} \frac{n^{\mu}}{P . n}\right) \\
& \epsilon^{(1) \mu}=-\frac{1}{\sqrt{\left(1-\xi^{2}\right)\left(t_{0}-t\right)}}\left((1+\xi) p^{\prime \mu}-(1-\xi) p^{\mu}\right. \\
&\left.\quad-\frac{\xi\left(t_{0}-t\right)-t_{0}}{\xi} \frac{n^{\mu}}{P . n}\right) \\
& \epsilon^{(2) \mu}= \frac{1}{\sqrt{\left(1-\xi^{2}\right)\left(t_{0}-t\right)}} \frac{2 \epsilon^{\mu}{ }_{\nu \alpha \beta} p^{\nu} p^{\alpha} n^{\beta}}{P . n}
\end{aligned}
$$

where $t_{0}=-4 M^{2} \xi^{2} /\left(1-\xi^{2}\right)$. The vectors $\epsilon(0)=\epsilon^{(0)}$ and $\epsilon( \pm 1)=\mp\left(\epsilon^{(1)} \pm i \epsilon^{(2)}\right) / \sqrt{2}$ then correspond to definite light-cone helicity [12]. This approximately coincides with usual helicity in frames where the deuteron moves fast, provided that $\operatorname{sgn}\left(p^{3}\right)=-\operatorname{sgn}\left(n^{3}\right)$. The polarizations for the outgoing deuteron are obtained from Eq. (17) by the exchange $p^{\mu} \leftrightarrow p^{\prime \mu}, \xi \leftrightarrow-\xi$ and an overall sign change for $\epsilon^{(1)}$ and $\epsilon^{(2)}$. With this we get

$$
\begin{aligned}
\mathcal{A}_{++,++} & =\frac{H_{1}}{2}-\frac{H_{5}}{6}+\frac{D H_{3}}{2}+\frac{\tilde{H}_{1}}{2}+2 D\left(\tilde{H}_{2}+\xi \tilde{H}_{3}\right), \\
\mathcal{A}_{0+, 0+} & =\frac{H_{1}}{2}-\xi H_{4}+\frac{1}{3}\left(1-\frac{3}{2} \xi^{2}\right) H_{5} \\
& -\left(D-\frac{\xi^{2}}{1-\xi^{2}}\right)\left(H_{1}-H_{2}-\xi H_{4}-\frac{1}{3} H_{5}\right)
\end{aligned}
$$

$$
\begin{aligned}
&-\left(D^{2}-\frac{\xi^{2}}{\left(1-\xi^{2}\right)^{2}}\right) H_{3} \\
& \mathcal{A}_{-+,++}=-D\left(\frac{1}{2} H_{3}+2\left(\xi \tilde{H}_{2}+\tilde{H}_{3}\right)\right) \\
& \mathcal{A}_{0+,++}=\sqrt{\frac{D(1-\xi)}{2(1+\xi)}}\left[H_{1}-\frac{1-\xi}{2}\left(H_{2}-H_{4}\right)\right. \\
&\left.-\frac{1}{3} H_{5}+\left(D-\frac{\xi}{1-\xi^{2}}\right) H_{3}\right]+\sqrt{2 D\left(1-\xi^{2}\right)}\left[\frac{1}{4}\left(\tilde{H}_{1}+(1-\xi) \tilde{H}_{4}\right)\right. \\
&\left.+\left(D-\frac{\xi}{1-\xi^{2}}\right)\left(\tilde{H}_{2}+\tilde{H}_{3}\right)\right]
\end{aligned}
$$

where $D=\left(t_{0}-t\right) /\left(4 M^{2}\right)$. The remaining amplitudes can be easily obtained from the relations (15), (16) and

$$
\mathcal{A}_{\lambda^{\prime}-\mu, \lambda-\mu}=\mathcal{A}_{\lambda^{\prime} \mu, \lambda \mu}\left(\tilde{H}_{i} \longrightarrow-\tilde{H}_{i}\right) .
$$

As required by angular momentum conservation, one gets a factor $\sqrt{t_{0}-t}$ for each unit of helicity flip. Note that $H_{2,4}$ and $\tilde{H}_{4}$ only appear with longitudinal deuteron polarization, and that the only GPDs appearing in double helicity flip amplitudes are $H_{3}$ and $\tilde{H}_{2,3}$. In the forward limit we have $\mathcal{A}(x, 0,0)_{\lambda+, \lambda+}=q_{\uparrow}^{\lambda}(x)$, $\mathcal{A}(x, 0,0)_{\lambda-, \lambda-}=q_{\downarrow}^{\lambda}(x)$ and find the relations (12).

Gluon distributions. Let us turn to the gluon distributions in the deuteron. Instead of the matrix elements (11), (2) we now have

$$
\begin{gathered}
4 \frac{n_{\alpha} n_{\beta}}{P . n} \int \frac{d \kappa}{2 \pi} e^{i x \kappa P . n}\left\langle p^{\prime}, \lambda^{\prime}\left|F^{\alpha \mu}(-\kappa n) F_{\mu}^{\beta}(\kappa n)\right| p, \lambda\right\rangle \\
=\sum_{i} \epsilon^{\prime * \beta} V_{\beta \alpha}^{(i)} \epsilon^{\alpha} H_{i}^{g}(x, \xi, t), \\
-4 i \frac{n_{\alpha} n_{\beta}}{P . n} \int \frac{d \kappa}{2 \pi} e^{i x \kappa P . n}\left\langle p^{\prime}, \lambda^{\prime}\left|F^{\alpha \mu}(-\kappa n) \tilde{F}_{\mu}^{\beta}(\kappa n)\right| p, \lambda\right\rangle \\
=\sum_{i} \epsilon^{\prime * \beta} A_{\beta \alpha}^{(i)} \epsilon^{\alpha} \tilde{H}_{i}^{g}(x, \xi, t),
\end{gathered}
$$

with $\tilde{F}^{\alpha \beta}=\frac{1}{2} \epsilon^{\alpha \beta \gamma \delta} F_{\gamma \delta}$. We take the same tensors $V^{(i)}, A^{(i)}$ as for quark distributions, given in Eq. (6). Note that the $H_{i}^{g}$ are even and the $\tilde{H}_{i}^{g}$ odd in $x$. Their behavior under time reversal is the same as in (9) for the corresponding quark distributions, and the definitions and expressions of the helicity amplitudes $\mathcal{A}_{\lambda^{\prime} \mu, \lambda \mu}^{g}$ are also analogous to the quark case. The forward limit is now $\mathcal{A}^{g}(x, 0,0)_{\lambda+, \lambda+}=x g_{\uparrow}^{\lambda}(x)$ and $\mathcal{A}^{g}(x, 0,0)_{\lambda-, \lambda-}=x g_{\downarrow}^{\lambda}(x)$, with an extra factor $x$ compared to the quark case.

Some Phenomenology. The deuteron GPDs can be accessed in hard exclusive processes such as deeply virtual Compton scattering in $e d \rightarrow e d \gamma$, and electroproduction $e d \rightarrow e d M$ of a meson or a meson pair [16]. The relevant kinematical limit is that of large invariant momentum transfer $Q^{2}$ to the lepton at fixed $x_{B}$ and $t$, where 
the Bjorken variable $x_{B}$ is defined as in deep inelastic scattering. Factorization formulae are the same as for nucleon targets [5, 13], with the appropriate replacement of the hadronic matrix elements, but the same hard scattering kernels. The $Q^{2}$ behavior of the amplitudes and the selection rules for photon and meson helicities also remain the same, since they depend on the hard-scattering process, not on the target spin. Electroproduction of a pseudoscalar meson selects the GPDs $\tilde{H}_{i}$, vector meson production involves the $H_{i}$ and $H_{i}^{g}$, and all distributions appear in Compton scattering. Notice that the isosinglet nature of the deuteron simplifies the flavor structure of the GPDs and thus of the scattering amplitudes. One consequence is that pion exchange does not contribute to any of these processes, in contrast to the nucleon case, where it may give important contributions through the quark distribution $\tilde{E}$ [14]. In kinematics where the BetheHeitler process dominates in electroproduction $e d \rightarrow e d \gamma$, one can use the methods of 15] to study Compton scattering through the interference of the two processes. At sufficiently large $Q^{2}$ this interference term gives access to a linear combination of GPDs, weighted with the electromagnetic deuteron form factors $G_{1,2,3}(t)$.

Discussion. To get a feeling for the physics of GPDs in the deuteron, consider the approximation where they are written as a convolution of the nucleon GPDs with the light-cone wave function $\psi_{p+n}$ for a proton and a neutron in the deuteron [17]. The struck nucleon then has to absorb the entire momentum transfer, in particular its plus-component parameterized by $\xi$. Assuming for simplicity that $\psi_{p+n}$ is only nonzero if the plus-momentum fraction of the proton in the deuteron is between $\frac{1}{2}(1-w)$ and $\frac{1}{2}(1+w)$, one finds that all $H_{i}$ and $\tilde{H}_{i}$ vanish for $\xi>w$. The $\xi$ dependence of the deuteron GPDs thus reflects the width of the wave function $\psi_{p+n}$ in longitudinal momentum fraction. For $\xi$ well above $w$ they provide access to deuteron wave function components that cannot be described in terms of individual nucleons. To unravel such components in inclusive deep inelastic scattering at $x_{B}>1$ has turned out to be difficult. Approaching this region from below, the struck quark has to take the entire momentum of a nucleon in the convolution picture, so that not only the deuteron wave function $\psi_{p+n}$ becomes small but also the parton density in the nucleon. This is not the case for GPDs with their independent momentum variables $x$ and $\xi$ : at $\xi>w$ one can still have any value for the plus-momentum fraction of the struck parton in the target.

We also note that, since in a convolution model $b_{1}$ requires a $d$ wave component in $\psi_{p+n}$ [1], the same holds for $H_{5}$. Double helicity flip amplitudes also need a $d$ wave admixture: the helicity flip of a nucleon cannot exceed one unit, so that orbital angular momentum is necessary. Hence $H_{3}$ and $\tilde{H}_{2,3}$ involve the $d$ wave part of $\psi_{p+n}$.

In this letter we have focused on the case where the deuteron scatters elastically. One may extend our study to the case where it dissociates into a proton and neutron, or a more complicated hadronic system, introducing appropriate transition GPDs. Factorization still holds in the processes discussed above, provided that the invariant mass of the dissociative system is small compared with the hard scale $Q^{2}$.

Let us finally stress that for low enough $\xi$ the generalized parton distributions for elastic deuteron transitions are by no means small. Neither is there any suppressing factor in the cross sections. It should thus be possible to perform exclusive electroproduction experiments on a deuteron target where those on a nucleon are possible. We hope for a rich harvest of physics on this topic in the forthcoming years at existing facilities such as DESY and Jefferson Lab.

Acknowledgments. We acknowledge discussions with J. P. Ralston and O. V. Teryaev. This work is supported in part by the TMR and IHP programmes of the European Union, Contracts No. HPRN-CT-2000-00130 and No. FMRX-CT98-0194. CPhT is UMR 7644 of CNRS.

[1] P. Hoodbhoy, R. L. Jaffe, A. Manohar, Nucl. Phys. B 312, 571 (1989).

[2] S. J. Brodsky, C. Ji, G. P. Lepage, Phys. Rev. Lett. 51, 83 (1983).

[3] M. Garçon, J. W. Van Orden, nucl-th/0102049

[4] D. Müller et al., Fortsch. Phys. 42, 101 (1994) thep$\mathrm{ph} / 9812448$;

X. Ji, Phys. Rev. Lett. 78, 610 (1997) hep-ph/9603249.

[5] A. V. Radyushkin, Phys. Rev. D 56, 5524 (1997) thep$\mathrm{ph} / 9704207$.

[6] B. de Wit, J. Smith, Field Theory in Particle Physics, Vol. I, Amsterdam (North Holland) 1986.

[7] T. Frederico et al., Phys. Rev. C 46, 347 (1992); R. G. Arnold, C. E. Carlson, F. Gross, Phys. Rev. C 23, 363 (1981).

[8] J. Blümlein, B. Geyer, D. Robaschik, Nucl. Phys. B560, 283 (1999) hep-ph/9903520.

[9] A. V. Belitsky, A. Freund, D. Müller, Nucl. Phys. B574, 347 (2000) hep-ph/9912379.

[10] M. Diehl, Eur. Phys. J. C 19, 485 (2001).

[11] F. E. Close, S. Kumano, Phys. Rev. D 42, 2377 (1990).

[12] S. J. Brodsky, H. Pauli, S. S. Pinsky, Phys. Rept. 301, 299 (1998).

[13] J. C. Collins, L. Frankfurt, M. Strikman, Phys. Rev. D 56, 2982 (1997) hep-ph/9611433;

J. C. Collins, A. Freund, Phys. Rev. D 59, 074009 (1999) hep-ph/9801262;

X. Ji, J. Osborne, Phys. Rev. D 58, 094018 (1998) hep$\mathrm{ph} / 9801260$.

14] K. Goeke, M. V. Polyakov, M. Vanderhaeghen, hep$\mathrm{ph} / 0106012$.

[15] M. Diehl, T. Gousset, B. Pire, J. P. Ralston, Phys. Lett. B 411, 193 (1997) hep-ph/9706344;

A. V. Belitsky, D. Müller, L. Niedermeier, A. Schäfer, 
Nucl. Phys. B 593, 289 (2001) hep-ph/0004059.

[16] M. Diehl et al., Phys. Rev. Lett. 81, 1782 (1998).

[17] L. L. Frankfurt, M. I. Strikman, Phys. Rept. 76, 215 (1981);

J. Carbonell et al., Phys. Rept. 300, 215 (1998) nuclth/9804029;

G. A. Miller, Prog. Part. Nucl. Phys. 45, 83 (2000) nuclth/0002059. 